\title{
Discussion on Measurement and Field Calibration of Digital Energy Meter
}

\author{
XIAO Ji, CHENG Yingying, DU Jie, ZHOU Feng \\ State Grid Chongqing Electric Power CO. Electric Power Research Institute
}

\begin{abstract}
Keywords: Digital energy meter; Fiber; Energy Measurement;
Abstract: Based on the measurement principle of digital energy meter, the measurement error of the digital energy meter of intelligent substation is mainly caused by the defect of the adopted algorithm, the incompleteness of the received data message and the effective number of the floating point. This paper expounds the necessity of carrying out on-site inspection of digital electric energy meter with real load, and explores the way of field test.
\end{abstract}

\section{Introduction}

As the measure device employed by power producer, power seller and power consumer, the plays a significant role in power system and has been widely used. Therefore, the safety, reliability and accuracy of the watt-hour meter needs to be guaranteed strongly. Traditional watt-hour meter that consists of current transformer, voltage transformer and electricity meter has some disadvantages of high power consumption, large error, material waste, electricity easily stolen and so on. With the great development of many techniques such as measuring sensor, communication, information technology, an advanced power meter has been developed. By setting the measuring circuit hanging on the HV side, this new type of power meter can prevent the electricity stolen. In this paper, to distinguish with the traditional watt-hour meter, this new type of watt-hour meter is called high voltage watt-hour meter. The high voltage watt-hour meter mainly bases on the micro controller to realize the measurement, data monitoring and communication. It has advantages of high intelligence and accuracy. To guarantee the stable operation of the high voltage meter, it is necessary for the micro controller and other integrated chip to obtain supply power at the status of high voltage. Currently, the power supply mode mainly contains method below.

With the general construction of intelligent substation, digital energy meter has been widely used as a new type of measurement equipment. The trend of diversified digital energy meter occurs according to the application needs. Because the measuring principle and the interface mode of the digital electric energy meter is different from the induction and the electronic electric energy meter, traditional field inspection technology has been unable to carry out the actual load test work. From the operating experience, domestic and foreign experts generally believe that the operation of digital power meters still need on-site inspection work to be carried out, to ensure the stability, accuracy and reliability of the metering equipment. At present, there is no uniform standard for the content of the on-site inspection technical indicators, standards and so on. Based on the measurement principle of digital meter, this paper summarizes the practical experience of engineering application and determines the basic test method of digital electric energy meter field test.

\section{Function structure and measurement principle of digital electric energy meter}

Digital electric energy meter is composed of data processing unit, communication unit and so on. Receive current and voltage sampling data message that matches DL/T860.92 frame format. After the protocol analysis, data calculation and processing, power measurement is achieved.

Electronic transformer outputs digital voltage and current signal, which is transmitted to merging unit through optical fiber. The merging unit combines the voltage and current information into the standard data frame according to the IEC61850-9 protocol standard. Digital electric energy meter 
receives the data frame, and the parameters such as electric energy can be calculated by mathematical calculation. The digital signal power measurement formula is:

$$
\begin{aligned}
& U_{i}=U_{m} \cos (\omega \cdot i / N) \\
& I_{i}=I_{m} \cos (\omega \cdot i / N+\varphi) \\
& E=T \sum_{i=1}^{N} U_{i} I_{i}
\end{aligned}
$$

$\omega$ represents power grid operating angle frequency; Um and Im represents the voltage and current signal amplitude respectively; $\mathrm{T}$ represents time interval of sampling for electronic instrument transformer (Common sampling rate is 80 points per cycle namely $4 \mathrm{kHz}, 200$ points per cycle namely $10 \mathrm{kHz}$ ); $\mathrm{N}$ represents sampling points in a sampling period.

Digital electric meter has advantages as follows:

1) High accuracy: Digital electric energy meter has no analog digital conversion unit, only digital receiving and electric energy metering algorithm, so it can obviously improve the accuracy of electric energy metering. At the same time, the digital electric energy metering device uses the optical fiber to transmit sampling value, and the transmission digital signal has no wire voltage drop. The elimination of lead pressure drop can improve the measurement accuracy of the whole electric power measurement system.

2) High reliability: The communication between the digital electric energy meter and the merging unit adopts the optical fiber to realize the complete electrical isolation, ensuring that the digital current and voltage signal transmission interference can not be caused under various complicated electromagnetic environment. The digital electric energy meter cancels the input of analog voltage and current signal, and effectively eliminates the hidden danger of the safety accidents such as over current or second current open circuit.

3) High stability: Digital electric energy meter adopts digital signal input and no analog sampling equipment. In the long run, it effectively avoids the influence of the traditional electric energy meter caused by the temperature, the resistance capacitance of the sampling circuit, the zero drift, the mechanical wear, the electromagnetic interference and so on.

\section{Analysis of error sources of digital electric energy meter}

\section{DC offset error analysis}

Active analog electronic devices are generally difficult to achieve full symmetry, so there will be a DC offset voltage or DC offset current output. The existing merging unit technical condition does not limit the DC offset of the merging unit, so the scene from the combined unit output DC offset is defaulted reasonable. When the load is low or the power factor is low, the effective value and active power will result in a certain error.

\section{Frame error analysis}

When the active power is calculated in a single window frame, the error caused is related to the sampling phase when packet loss occurs and the numbers of sampling points of each cycle $\mathrm{N}$ and the calculation of the sampling period number $n$.

\section{Numerical integration error analysis}

In the actual power system, the frequency and amplitude of the current and voltage changes with time, and the pure sine signal is nonexistent, especially in the case of abnormal power supply, such as electric drive, electric furnace smelting, which will make the current and voltage have relatively large fluctuations. So in the laboratory of digital input type of electric energy meter, the impact of frequency 
should be increased. At the same time, the digital signal source used in the experiment should be converted from the actual analog quantity through A/D. Otherwise, the electric energy meter which uses the first order point product and integral algorithm can result in the difference between the actual operation error and the error of the laboratory test.

\section{Error analysis caused by asynchronous sampling}

\section{Other error}

The algorithm error sources of digital input electric energy meter are many aspects. For instance, point to point communication interpolation error, sampling rate error, sampling point error; Some errors are related to each other, some are independent, and some errors can be ignored. Energy meter manufacturers should use the appropriate digital filter, appropriate numerical integral and interpolation algorithm and appropriate design of the program to ensure the error of digital input electric energy meter in the laboratory and field.

\section{Field inspection technology of digital electric energy meter}

\section{Test necessity}

The digital electric energy meter receives the digital voltage and the current quantity which is sent by the merging unit. In theory, there is no error in the processing of digital voltage and current. In fact, due to the use of the algorithm may be defective, the received voltage and current of data packets may not be complete. And because of the effective number of floating point numbers, digital watt hour meter can generate error.

\section{Principle of field load test}

The merging unit of the field photoelectric transformer sends message to the digital electric energy meter and the digital electric energy meter checking instrument through the IEC61850 protocol digital signal according to the actual measured value of the field. According to the algorithm, digital electric energy meter can generate electric energy pulse M1 and digital electric energy meter also bases on the protocol and algorithm to generate pulse M2. Then contrast M2 and M1 to calculate the actual power error.

\section{Field inspection technology}

a.Two ways of connection :

1) There is alternate network port on site

As the parallel connection of digital signals can be realized only through the redundant network ports, the test can be done only by using the standby network port to access the digital input standard electric energy meter and collecting the pulse output of the digital input type electric energy meter with the help of photoelectric.

2) There is no spare optical fiber

The combined unit is connected with the optical fiber of the electric energy meter to pull out the access field calibration instrument, and a set of optical fiber to the electric energy meter is drawn out from the calibration instrument. Optical signal indicating lamp's lighting means switching on, the signal lights flashing light means data exchange exists. After optical fiber connection is completed, connect the light pulse line.

b.Check parameter acquisition and setting :

The voltage and current channel can be obtained by the following three methods:

1) Gets the configuration file when the new substation is accepted.This method is the most reliable, but note the difference between the configuration file and the channel number of the start bit ( 0 or 1 start) 
2) Part of the digital table can be displayed by the button LCD screen, but pay attention to the 16 and 10 hex conversion.

3) Obtain by reading message information

c.Parameter setting :

Digital electric energy meter field load test generally need to do the following settings on the calibration instrument:

Connection mode; pulse input channel; MAC address; measured constant; pulse input channel number; protocol type; sampling; ASDU / frame; metering channel; PT/CT ratio etc.

\section{Conclusion}

At present, the digital power meter field inspection procedures has not yet been issued by state or industry. But according to the principle of electric energy meter it can be inspected. According to the requirements of the IEC61850-9 protocol, the output pulse of the digital meter is compared with that of the standard pulse, and the error of electric energy meter can be obtained. All aspects of the digital electric energy meter verification method need to be standardized and improved. Generally speaking, the digital electric energy meter can solve many inherent defects of the traditional electric energy meter, and display the superiority in the aspect of data accuracy and reliability. But there are new problems. For example, it relies on external work power, once power is lost, then metering is stopped. Due to the input of the digital signal, signal channel stability, switch and interface quality, signal packet loss and other issues can not be ignored. Further research is needed in the measurement principle and test mode to ensure that the digital electric energy meter can meet the measurement requirements of electric power system.

\section{References}

[1] Wei Mingsun, Digital electric energy measurement technology, China Electric Power Press , 2014.

[2] Technical management regulation of electric energy metering device, DL/448-2000

[3] Zhang Linqing, Analysis on electric energy metering device abnormal real-line monitoring[J],China Electric Power,2012(4):37-39.

[4] Wang Chunqing, The analysis of present operational situation for electrical energy metering device[J],Electrical Measurement \& Instrumentation,2010(7):26-28. 